\title{
MULTIWAVELENGTH TEMPORAL BEHAVIOR OF GRS 1915+105
}

\author{
D. HANNIKAINEN \\ Observatory, $P O$ Box 14 \\ 00014 University of Helsinki, Finland \\ AND \\ PH.DUROUCHOUX \\ C.E. Saclay, DSM, DAPNIA, Service d'Astrophysique \\ 91191 Gif sur Yvette, Cedex France
}

\section{GRS $1915+105$}

The transient X-ray source GRS 1915+105 was discovered in August 1992 with the GRANAT/WATCH all-sky monitor (Castro-Tirado et al. 1994). Subsequent VLA observations from March through April 1994 led to the discovery of apparent superluminal motion in a pair of radio condensations moving away from the compact radio core (Mirabel \& Rodriguez 1994). These jet-like features are interpreted as a bipolar outflow with bulk velocity $\sim 0.9 \mathrm{c}$. Although no optical counterpart has been observed, due to the heavy extinction in the Galactic plane, and therefore not enabling measurements of the mass of the compact object, the hard X-ray spectrum and high luminosity $\left(\sim 10^{39} \mathrm{erg} \mathrm{s}^{-1}\right)$, extreme variability in the X-ray light curve and the relativistic jets make GRS $1915+105$ a strong black hole candidate.

GRS $1915+105$ has been extensively observed in the radio, X-ray and hard $\mathrm{X}$-ray since its discovery. In all wavebands the emission is highly variable and complex, including very large amplitude flaring on timescales of minutes in the X-rays (e.g. Greiner, Morgan \& Remillard 1996) and Pooley \& Fender (1997) have identified three different types of behavior in the radio flux density following a one and a half years' monitoring at $15 \mathrm{GHz}$ with the Ryle telescope. In Figure 1 we have gathered together a sample of light curves of GRS 1915+105, demonstrating flaring activity and periods of total quiescence in both the radio and X-ray domains. There is no obvious correlation between the radio and X-ray/gamma-ray behavior. 


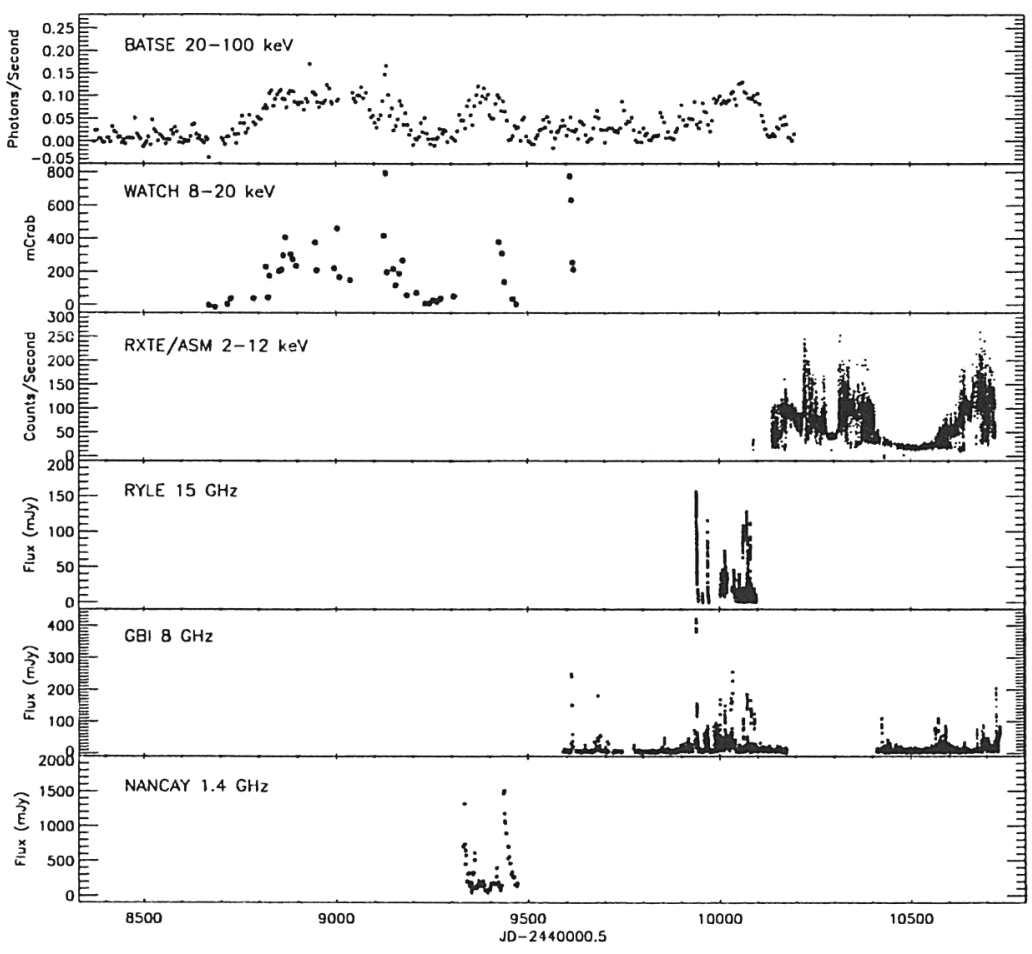

Figure 1. References. BATSE: http://cossc.gsfc.nasa.gov/cossc/batse/hilev/occ.html; WATCH: Finoguenov et al. (1994); RXTE: http://space.mit.edu/XTE/ASM_lc.html; RYle: R. Fender (priv. comm.); GBI: E. Waltman (priv. comm.) and http://www.gb.nrao.edu/gbint/GBINT.html; NANCAY: Rodriguez et al. (1995)

\section{Acknowledgements.}

The authors would like to thank Robert Fender, Craig Robinson, Elizabeth Waltman, William Heindl and Chris Schrader for their help in gathering the data. Public domain data from the NSF-NRAO-NASA Green Bank Interferometer. DH travelled to Kyoto on a grant from the Chancellor of the University of Helsinki.

\section{References}

Castro-Tirado, A. J. et al. 1994, ApJS 92, 469

Finoguenov, A. et al. 1994, ApJ 424, 940

Greiner, J., Morgan, E.H. \& Remillard, R. A. 1996, ApJ 473, L107

Mirabel, I. F. \& Rodriguez, L. F. 1994, Nature 371, 46

Pooley, G. \& Fender, R. 1997, MNRAS, submitted

Rodriguez, L. F. et al. 1995, ApJS 101, 173 